\title{
SOLVENTES EUTÉTICOS NATURAIS PROFUNDOS (NADES) NO PREPARO DE AMOSTRAS DE ROCHA FOSFÁTICA E SUPLEMENTO MINERAL PARA DETERMINAÇÃO ELEMENTAR POR TÉCNICAS DE PLASMA
}

\author{
Ana P. R. Santana ${ }^{a}$, Daniel F. Andrade ${ }^{\mathrm{b}}$, Taciana G. S. Guimarães ${ }^{\mathrm{a}}$, Clarice D. B. Amaral ${ }^{\mathrm{c}}$, Andrea Oliveira ${ }^{\mathrm{c}, *, \bullet}$, Ana R. A. \\ Nogueira $^{\text {d }}$ Mario H. Gonzalez ${ }^{\mathrm{a}}$ \\ aDepartamento de Química e Ciências Ambientais, Instituto Nacional de Tecnologias Alternativas para Detecção, Avaliação \\ Toxicológica e Remoção de Contaminantes Emergentes e Radioativos, Universidade Estadual Paulista "Júlio de Mesquita Filho", \\ 15054-000 São José do Rio Preto - SP, Brasil \\ bDepartamento de Química, Universidade Federal de São Carlos, 13565-905 São Carlos - SP, Brasil \\ 'Departamento de Química, Universidade Federal do Paraná, 81531-980 Curitiba - PR, Brasil \\ ¿Embrapa Pecuária Sudeste, 13560-970 São Carlos - SP, Brasil
}

Recebido em 11/10/2020; aceito em 26/01/2021; publicado na web em 24/02/2021

\begin{abstract}
NATURAL DEEP EUTECTIC SOLVENTS (NADES) IN SAMPLE PREPARATION OF PHOSPHATE ROCK AND MINERAL SUPPLEMENT FOR ELEMENTARY DETERMINATION BY PLASMA-BASED TECHNIQUES. Natural deep eutectic solvents (NADES) based on xylitol, citric acid, and malic acid were synthesized and used in ultrasound-assisted extraction (UAE) and heating-bath extraction of phosphate rock and mineral supplement samples. Arsenic, $\mathrm{Ca}, \mathrm{Cd}, \mathrm{Cr}, \mathrm{Cu}, \mathrm{Fe}, \mathrm{K}, \mathrm{Mg}, \mathrm{Mn}, \mathrm{Na}, \mathrm{P}$ and $\mathrm{Zn}$ were determined by inductively coupled plasma mass spectrometry (ICP-MS) and inductively coupled plasma optical emission spectrometry (ICP OES). The results showed the NADES as promising solvents for extraction of inorganic matrices compared to reference values and concentrations obtained using microwave-assisted acid digestion (MW-AD). Significant differences were observed for some elements, suggesting different chemical interactions between the synthesized NADES and each monitored element. For analytes extraction of phosphate rock, UAE presented the best results for As, $\mathrm{Cr}$ and $\mathrm{P}$. Zinc was extracted with UAE and heating bath. Concerning mineral supplement, no significant differences were observed for Ca (UAE), Cd (UAE and heating bath), K (UAE and heating bath), Mn (UAE and heating bath), Na (UAE and heating bath), and P (UAE and heating bath). The NADES is a greener and a potentially alternative for the sample preparation compared to the official methods of analysis, can being used as selective extractor solvent in conventional sample preparation methods.
\end{abstract}

Keywords: NADES; sample preparation; green chemistry; inorganic composition; inorganic samples.

\section{INTRODUÇÃO}

Em procedimentos experimentais como digestão ou extração, os solventes apresentam um papel crucial na etapa de preparo de amostra por meio da dissolução de sólidos, transferência de massa e extração de analitos. ${ }^{1}$ Portanto, há um grande interesse nos estudos para o desenvolvimento de novos solventes verdes, visando principalmente a substituição de solventes considerados voláteis e de elevada inflamabilidade e toxicidade. ${ }^{1,2}$

Considerando a determinação elementar de nutrientes e contaminantes em fertilizantes e suplementos minerais, a maioria dos métodos analíticos inclui algum tratamento da amostra. $\mathrm{O}$ preparo de amostra é uma etapa de extrema importância em um procedimento analítico, pois perdas dos analitos ou contaminações podem comprometer a precisão e a exatidão do resultado final.,

Os solventes eutéticos profundos (DES) são formados por um composto receptor de ligação de hidrogênio (HBA), com um composto doador de ligação de hidrogênio (HBD) dando origem a uma mistura que forma um sistema eutético, com ponto de fusão abaixo do apresentado por cada precursor individualmente. ${ }^{5}$ Quando os compostos que constituem os DES são aminoácidos, ácidos orgânicos, açúcares, ureia ou derivados de colina, os DES são denominados de solventes eutéticos naturais profundos (NADES). ${ }^{6,7}$ Durante a síntese dos NADES (e DES) não há qualquer solvente adicional ou formação de subprodutos, resultando em um material facilmente biodegradável. ${ }^{8}$

*e-mail: andrea.oliveira@ufpr.br
Os NADES oferecem vantagens como baixa toxicidade, biodegradabilidade, estabilização de soluto, sustentabilidade, preparo simples e viscosidade e densidade ajustáveis. ${ }^{9}$ Em geral, combinações de cloreto de colina e seus derivados (receptor de ligação de hidrogênio) com glucose, xilitol, ácido cítrico, ácido málico, ácido oxálico, ácido tartárico, ácido propanoico e frutose, atuando como doadores de ligação de hidrogênio, são os principais exemplos relatados na literatura sobre síntese dos NADES. ${ }^{10}$

A digestão ácida assistida por radiação micro-ondas (MW-AD) é um dos procedimentos mais utilizados na etapa de preparo de amostra, e pode ser utilizado como método padrão para estudos de preparo de amostra. ${ }^{11-14} \mathrm{~A}$ extração assistida por radiação ultrassônica (UAE) tem sido utilizada para o preparo de amostra. As ondas acústicas, com frequência entre 20 e $1000 \mathrm{kHz}$, ocasionam a agitação e o aquecimento do meio, podendo gerar o fenômeno de cavitação, que envolve a nucleação, o crescimento e a implosão de microbolhas em líquidos. ${ }^{15} \mathrm{O}$ colapso das bolhas durante a irradiação de um líquido em um campo ultrassônico resulta em perturbações que elevam a temperatura e a pressão localizadas, produzindo microjatos. ${ }^{16}$ Esses por sua vez causam erosão na amostra sólida e auxiliam na extração dos analitos, assim como o aumento da temperatura. ${ }^{16}$ Além disso, o efeito de homogeneização intensa gerado pela propagação do ultrassom no meio líquido contribui para o aumento da transferência de massa, melhorando de forma significativa a taxa de transferência. ${ }^{17}$ No geral, mecanismos de cavitação, fragmentação, erosão, efeito sonoro-capilar, sonoporação, estresse de cisalhamento local e destruição de texturação agem na UAE, cuja eficiência provavelmente ocorre devido à uma combinação desses efeitos. ${ }^{17}$ Procedimentos de 
UAE foram utilizados nos mais diferentes tipos de amostra como solo, sedimento, vegetal, tecido animal e lama empregando soluções em meio ácido. ${ }^{18-21}$

Os fertilizantes constituem uma importante fonte de nutrientes essenciais para as plantas e solo. No entanto, fertilizantes fosfatados podem conter elementos potencialmente tóxicos, originários da rocha ou da matéria-prima empregada para sua industrialização. ${ }^{22}$ Esses elementos podem ser absorvidos pelas plantas e ingressar na cadeia alimentar. ${ }^{23}$ Estima-se que as rochas fosfatadas usadas na produção de fertilizantes são as maiores fontes de contaminação de $\mathrm{Cd}$ em solos agrícolas. ${ }^{24} \mathrm{O}$ conhecimento da concentração dos elementos em rochas fosfatadas é fundamental, devido ao seu uso como matéria prima para a produção de fertilizantes fosfatados.

A suplementação animal garante os níveis adequados de elementos essenciais, tais como $\mathrm{Ca}, \mathrm{Cu}, \mathrm{Fe}, \mathrm{K}, \mathrm{Na}, \mathrm{Mg}, \mathrm{P}$ e $\mathrm{Zn}$, evitando que sua deficiência cause alterações nas funções metabólicas dos animais. ${ }^{25}$ Por exemplo, o Ca é importante para a formação dos ossos e manutenção dos músculos, enquanto que o fósforo apresenta uma função importante na formação dos fosfolipídios e na microbiota do rúmen em ruminantes. ${ }^{25,26}$ Elementos como $\mathrm{Cu}, \mathrm{Fe}, \mathrm{Mg}$ e $\mathrm{Zn}$ são encontrados na estrutura de certas enzimas que são indispensáveis em várias vias bioquímicas. ${ }^{25}$ Além disso, estudos apontam que o emprego da suplementação animal tem apresentado um aumento na porcentagem de nascimento e na taxa de crescimento e redução do índice de mortalidade em bovinos. ${ }^{26}$

Entre os macronutrientes e micronutrientes presentes em suplementos minerais, há pelo menos 15 minerais que são essenciais para a nutrição dos bovinos. ${ }^{27}$ Entretanto, estudos mostraram que elementos potencialmente tóxicos presentes em suplementos minerais para animais podem ocasionar alterações de ordem reprodutiva. ${ }^{26,28}$ Diante disso, torna-se necessária a análise elementar de fertilizantes e suplementos minerais antes do uso, para verificação da composição química e para o controle da contaminação desses produtos.

Devido à capacidade de atuar como agentes de estabilização, juntamente com suas habilidades como solventes, os NADES são uma alternativa interessante para a etapa de preparo das amostras. ${ }^{10}$ A maior parte das aplicações dos NADES está relacionada à extração de analitos orgânicos como ácidos fenólicos, hidrocarbonetos policíclicos aromáticos (HPAs), flavonoides e compostos fenólicos. ${ }^{29}$

Atualmente há poucos trabalhos na literatura que relatam o uso de NADES ou DES para a extração de analitos inorgânicos. ${ }^{29-31}$ NADES a base de ácido cítrico, ácido málico e xilitol foram utilizados em UAE para a extração de As, $\mathrm{Ca}, \mathrm{Cd}, \mathrm{Cu}, \mathrm{Fe}, \mathrm{K}, \mathrm{Mg}, \mathrm{Mn}, \mathrm{Na}, \mathrm{P}$ e $\mathrm{Zn}$ em amostras vegetais. ${ }^{30}$ NADES foram também utilizados em procedimentos de UAE e extração assistida por radiação microondas (MAE) para a extração de $\mathrm{As}, \mathrm{Cd}, \mathrm{Hg}, \mathrm{Pb}, \mathrm{Se}$ e V em tecidos biológicos e amostras vegetais. ${ }^{31}$

O objetivo deste trabalho foi a aplicação de dois NADES com diferentes combinações de ácido cítrico, ácido málico e xilitol empregando extração assistida por ultrassom (UAE) e extração por banho de aquecimento para determinação de $\mathrm{As}, \mathrm{Ca}, \mathrm{Cd}, \mathrm{Cr}, \mathrm{Cu}$, $\mathrm{Fe}, \mathrm{K}, \mathrm{Mg}, \mathrm{Mn}, \mathrm{Na}, \mathrm{P}$ e Zn em matrizes inorgânicas (fosfatado de rocha e suplemento mineral) por espectrometria de massas com plasma acoplado indutivamente (ICP-MS) e espectrometria de emissão atômica com plasma acoplado indutivamente (ICP OES). Entre as diversas características importantes dessas técnicas, está o fato de serem multielementares e apresentarem ampla faixa linear e boas sensibilidade e precisão. Além dessas, o ICP-MS oferece os baixos limites de detecção (LOD) como principal característica. $^{32}$

\section{PARTE EXPERIMENTAL}

\section{Reagentes e amostras}

Água ultrapura em sistema de ultra purificação $(18 \mathrm{M} \Omega \mathrm{cm}$ ICW-3000, Merck KGaA, Darmstadt, Alemanha) foi usada em todas as extrações e no preparo de todas as soluções. As vidrarias utilizadas nos experimentos foram previamente descontaminadas em banho ácido contendo $10 \%\left(\mathrm{v} \mathrm{v}^{-1}\right)$ de $\mathrm{HNO}_{3}$ por $48 \mathrm{~h}$, seguido de lavagem completa com água. $\mathrm{O}$ procedimento de digestão ácida assistida por radiação micro-ondas (MW-AD) foi realizado com $\mathrm{HNO}_{3}$ (SigmaAldrich, MO, EUA) previamente purificado usando um sistema de destilação abaixo do ponto de ebulição (sub-CLEAN PTFE, Milestone, BG, Itália) e 30\% ( $\left.\mathrm{m} \mathrm{v}^{-1}\right) \mathrm{H}_{2} \mathrm{O}_{2}$ (Sigma-Aldrich, MO, EUA). Soluções padrão para as curvas de calibração foram preparadas a partir de $1000 \mathrm{mg} \mathrm{L}^{-1}$ de soluções estoque monoelementares dos elementos químicos As, Ca, Cd, Cr, Cu, Fe, K, Mg, Mn, Na, P e Zn (Sigma-Aldrich, MO, EUA) por diluições consecutivas.

Para a síntese dos NADES foram utilizados ácido cítrico (SigmaAldrich, 99,5\% de pureza), DL-ácido málico (Sigma-Aldrich, 99\% de pureza), xilitol (Sigma-Aldrich, 99\% de pureza), todos na forma de pó cristalino branco, e água. A exatidão do método foi avaliada usando materiais de referência de suplemento mineral (RM-Agro E2001a) e fosfato de rocha (RM-Agro E2003a), produzidos pela Empresa Brasileira de Pesquisa Agropecuária (Embrapa). ${ }^{33,34}$

Os seguintes equipamentos foram empregados para o preparo das amostras e determinações: agitador magnético (LAbNet, agitador AccuPlate Hotplate, Edison, NJ, EUA), balança analítica (GEHAKA, Modelo AG200, São Paulo, SP, Brasil), forno de micro-ondas (Multiware PRO - Anton Paar GmbH, Graz, Austria), banho de aquecimento (EVLAB, Modelo B.M.EV:O15, Londrina, PR, Brasil), banho ultrassônico (Eco-Sonics, Modelo Q3.0/40 A, com frequência de $40 \mathrm{kHz}$, Indaiatuba, SP, Brasil). O ICP OES (iCAP 7000, Thermo Fisher Scientific, Madison, WI, EUA) e ICP-MS modelo NexION 300X (Perkin-Elmer, Shelton, CT) foram utilizados para as determinações dos analitos.

\section{Síntese dos NADES}

Dois tipos diferentes de NADES foram preparados com base no método apresentado por Santana et al. ${ }^{35}$ Os NADES foram preparados pelas combinações dos componentes: ácido cítrico, ácido málico e água (AC-AM) e ácido cítrico, xilitol e água (AC-Xil), com razão molar 1:1:10 de cada componente, respectivamente. As misturas foram colocadas em um béquer tampado com um vidro de relógio e aquecido em banho-maria por 2 horas a $50{ }^{\circ} \mathrm{C}$ sob agitação magnética na velocidade de $220 \mathrm{rpm}$. Foram sintetizados aproximadamente 100 $\mathrm{mL}$ de cada solvente, volume suficiente para os estudos realizados. Posteriormente, os solventes foram armazenados em dessecadores em temperatura ambiente para evitar a variação das propriedades físicas como densidade e viscosidade. ${ }^{36}$

A densidade dos NADES foi determinada de forma volumétrica utilizando picnômetro calibrado com água a $24{ }^{\circ} \mathrm{C}$ e balança analítica com precisão de $\pm 0,0001 \mathrm{~g} .{ }^{35} \mathrm{~A}$ viscosidade dos solventes foi determinada empregando um viscosímetro Cannon-Fenske calibrado com água a $24{ }^{\circ} \mathrm{C} .{ }^{35}$ As propriedades físico-químicas foram determinadas em triplicata.

\section{Extração assistida por ultrassom (UAE) com NADES}

Aproximadamente $100 \mathrm{mg}$ de amostras de suplemento mineral (RM-Agro E2001a) e fosfato de rocha (RM-Agro E2003a), foram pesados diretamente em frascos de $50 \mathrm{~mL}$. A seguir foram adicionados 
9,0 $\mathrm{mL}$ de cada um dos NADES (preparados individualmente) e a mistura submetida a banho ultrassônico por 45 min. Após o tempo de extração, adicionou-se $4 \mathrm{~mL}$ de água e o extrato foi centrifugado a $4000 \mathrm{rpm}$ por 5 minutos. Após a centrifugação, o sobrenadante foi separado do resíduo por filtração, visando evitar o contato entre o sólido e o extrato. O sobrenadante foi analisado por ICP OES e ICP-MS em até $48 \mathrm{~h}$ para as devidas determinações. Toda etapa de extração foi realizada em triplicata. ${ }^{30}$

\section{Extração por banho de aquecimento com NADES}

Aproximadamente $100 \mathrm{mg}$ das amostras de suplemento mineral (RM-Agro E2001a) e fosfato de rocha (RM-Agro E2003a) foram pesadas diretamente em frascos de $50 \mathrm{~mL}$, e adicionados $9,0 \mathrm{~mL}$ de cada um dos NADES (sintetizados individualmente). A mistura resultante foi submetida a banho de aquecimento na temperatura de $60{ }^{\circ} \mathrm{C}$ por $45 \mathrm{~min}$, sem agitação. Após a etapa de extração, adicionaram-se $4 \mathrm{~mL}$ de água e o extrato foi centrifugado a $4000 \mathrm{rpm}$ por $5 \mathrm{~min}$. Após a centrifugação, o sobrenadante foi separado do resíduo por filtração para evitar o contato entre o sólido e o extrato. O sobrenadante foi analisado por ICP OES e ICP-MS em até $48 \mathrm{~h}$ para as devidas determinações. Toda etapa de extração foi realizada em triplicata.

\section{Digestão ácida assistida por radiação micro-ondas (MW-AD)}

Digestão ácida assistida por radiação micro-ondas (MW-AD) foi empregada como método comparativo para a determinação dos teores dos elementos analisados pelos métodos de extração empregando NADES. As digestões foram realizadas em um forno micro-ondas, baseado no método descrito para a produção do material de referência de fosfato de rocha ${ }^{34}$ e por Kane and Hall (2016). ${ }^{37}$ Aproximadamente $200 \mathrm{mg}$ de amostras foram digeridos em frascos fechados com $6 \mathrm{~mL}$ ( $\left.7 \mathrm{~mol} \mathrm{~L}^{-1}\right)$ de ácido nítrico e $2 \mathrm{~mL}$ de $\mathrm{H}_{2} \mathrm{O}_{2}\left(30 \% \mathrm{~m} \mathrm{v}^{-1}\right)$, empregando o seguinte programa de aquecimento: I) rampa de aquecimento até $190{ }^{\circ} \mathrm{C}$ por $20 \mathrm{~min}$; II) permanência a $190{ }^{\circ} \mathrm{C}$ por $20 \mathrm{~min}$ e III) resfriamento até $50{ }^{\circ} \mathrm{C}$ por $10 \mathrm{~min}$. Após a digestão, as amostras e os brancos tiveram seus volumes ajustados quantitativamente com água.

\section{Determinação de $\mathrm{Ca}, \mathrm{Cu}, \mathrm{Fe}, \mathrm{K}, \mathrm{Mg}, \mathrm{Mn}, \mathrm{Na}, \mathrm{P}$ e $\mathrm{Zn}$ por espectrometria de emissão óptica com plasma acoplado indutivamente (ICP OES)}

Para serem analisados por ICP OES os extratos foram diluídos no intervalo de 100 a 250 vezes. Na Tabela 1 são apresentados os parâmetros instrumentais e as linhas de emissão selecionadas para determinação de cada analito monitorado. A curva de calibração para as análises dos extratos foi realizada em meio de NADES $1 \%\left(\mathrm{v} \mathrm{v}^{-1}\right)$, enquanto que a curva de calibração para as análises dos digeridos foi realizada em meio de ácido nítrico $1 \%\left(\mathrm{v} \mathrm{v}^{-1}\right)$. Esses valores foram determinados considerando as diluições finais.

\section{Determinação de As, Cd e Cr por espectrometria de massas com plasma acoplado indutivamente (ICP-MS)}

Os extratos anteriormente foram diluídos no intervalo de 100 a 250 vezes para serem analisados por ICP-MS. O sistema de introdução de amostra é equipado com nebulizador concêntrico, uma câmara ciclônica e uma tocha de quartzo com um tubo injetor de quartzo (2,0 mm d.i.). Os parâmetros instrumentais para o ICP-MS estão descritos na Tabela 2 e a análise foi realizada no modo de colisão com discriminação por energia cinética (KED) como estratégia de correção a base de gás hélio para evitar possível interferência poliatômica para
Tabela 1. Parâmetros instrumentais para as análises por ICP OES

\begin{tabular}{|c|c|}
\hline Parâmetros Instrumentais & Condições de Operação \\
\hline $\begin{array}{l}\text { Potência de radiofrequência (RF) } \\
\text { aplicada }(\mathrm{kW})\end{array}$ & 1,15 \\
\hline $\begin{array}{l}\text { Tempo de integração de baixos } \\
\text { comprimentos de onda (s) }\end{array}$ & 15 \\
\hline $\begin{array}{l}\text { Tempo de integração de altos } \\
\text { comprimentos de onda (s) }\end{array}$ & 5 \\
\hline $\begin{array}{l}\text { Vazão de introdução da amostra } \\
\left(\mathrm{mL} \min ^{-1}\right)\end{array}$ & 2,1 \\
\hline Tempo de estabilização (s) & 5 \\
\hline Vazão do gás auxiliar $\left(\mathrm{L} \mathrm{min}^{-1}\right)$ & 0,5 \\
\hline Vazão do gás de nebulização $\left(\mathrm{L} \mathrm{min}^{-1}\right)$ & 0,7 \\
\hline Vazão do gás do plasma $\left(\mathrm{L} \mathrm{min}^{-1}\right)$ & 12 \\
\hline Replicatas & 3 \\
\hline $\begin{array}{l}\text { Elementos e linhas de emissão } \\
\text { monitorados (nm) }\end{array}$ & $\begin{array}{c}\text { Ca II } 396,847^{\mathrm{b}}, \text { Cu I } 327,396^{\mathrm{a}} \text {, Fe II } \\
238,204^{\mathrm{a}}, \text { K I } 766,490^{\mathrm{b}}, \\
\text { Mg II } 280,270^{\mathrm{a}}, \text { Mn II } 259,373^{\mathrm{a}}, \\
\text { Na I } 589,592^{\mathrm{b}}, \text { P I } 177,495^{\mathrm{a}}, \mathrm{e} \\
\text { Zn I } 213,856^{\mathrm{a}}\end{array}$ \\
\hline
\end{tabular}

${ }^{a}$ Modo de visão axial. I - linha atômica. ${ }^{\text {b} M o d o ~ d e ~ v i s a ̃ o ~ r a d i a l . ~ I I ~-~ l i n h a ~ i o ̂ n i c a . ~}$

Tabela 2. Parâmetros instrumentais para as análises por ICP-MS

Parâmetros Instrumentais

Potência de radiofrequência (W)

1600

Vazão do gás do plasma $\left(\mathrm{L} \mathrm{min}^{-1}\right)$

18

Vazão do gás auxiliar $\left(\mathrm{L} \mathrm{min}^{-1}\right)$

1,2

Vazão do gás de nebulização $\left(\mathrm{mL} \mathrm{min}^{-1}\right)$

0,7

Voltagem de entrada (V)

$-3,00$

Voltagem de saída (V)

$-32,00$

$\mathrm{CRO}^{\mathrm{a}}(\mathrm{V})$

$-15,00$

$\mathrm{QRO}^{\mathrm{b}}(\mathrm{V})$

$-12,00$

Parâmetros do Método

Número de varreduras por leitura (varreduras) 50

Leituras (replicatas)

Número de leituras (leituras) 3

Tempo de espera (s)

25

Gás de colisão

$\mathrm{He}$

$\mathrm{RPq}^{\mathrm{c}}(\mathrm{V})$

0,25

Vazão do gás da cela de colisão $\left(\mathrm{mL} \min ^{-1}\right)$

Intervalo de calibração $\left(\mu \mathrm{g} \mathrm{L}^{-1}\right)$

$0,1-15$

Isótopos analisados

${ }^{75} \mathrm{As}^{+},{ }^{111} \mathrm{Cd}^{+},{ }^{52} \mathrm{Cr}^{+}$

${ }^{a} \mathrm{CRO}$ (Cell Rod Offset): voltagem aplicada ao quadrupolo da cela de colisão. ${ }^{\mathrm{b}} \mathrm{QRO}$ (Quadrupole Rod Offset): voltagem aplicada ao quadrupolo analisador de massas. ${ }^{\mathrm{C}} \mathrm{R}$ Pq: está relacionado a voltagem aplicadas hastes do quadrupolo.

${ }^{75} \mathrm{As}^{+}\left({ }^{40} \mathrm{Ar}^{35} \mathrm{Cl}^{+}\right)$e para ${ }^{52} \mathrm{Cr}^{+}\left({ }^{40} \mathrm{Ar}^{12} \mathrm{C}^{+}\right)$. A curva de calibração para as análises dos extratos foi realizada em meio de NADES $1 \%\left(\mathrm{v} \mathrm{v}^{-1}\right)$, enquanto a curva de calibração para as análises dos digeridos foi realizada em meio de ácido nítrico $1 \%\left(\mathrm{v} \mathrm{v}^{-1}\right)$, concentração próxima a dos extratos após diluídos.

\section{RESULTADOS E DISCUSSÃO}

Após a síntese, os NADES devem apresentar-se como solventes líquidos, homogêneos e estáveis, sem a formação de precipitado em temperatura ambiente e pressão atmosférica, ${ }^{36}$ o que os tornam 
adequados para o uso como solventes na etapa de preparo de amostra. ${ }^{10}$ Além disso, a ocorrência de interações intermoleculares, envolvendo ligações de hidrogênio, é uma característica da formação de NADES, já evidenciada em trabalhos anteriores. ${ }^{30,35}$

A adição de água na síntese dos NADES ocasiona uma diminuição nos valores de densidade e viscosidade devido ao enfraquecimento dessas interações intermoleculares. ${ }^{38}$ Viscosidade e densidade são características físico-químicas importantes que influenciam a eficiência de extração. ${ }^{39}$ Baixos valores de densidade e viscosidade aumentam a difusividade, ocasionando um maior movimento das moléculas e consequentemente aumentando a eficiência de extração. ${ }^{40,41}$ Os valores de densidade determinados foram 1,3260 $\pm 0,0002$ e 1,3130 $\pm 0,0001 \mathrm{~g} \mathrm{~mL}^{-1}$ e viscosidade de 9,24 $\pm 0,06$ e $11,09 \pm 0,21 \mathrm{mPa} . \mathrm{s}$, ambos para NADES AC-AM e NADES AC-Xil, respectivamente, a $24{ }^{\circ} \mathrm{C} .{ }^{30}$

Considerando a determinação elementar por técnicas de plasma (i.e. ICP OES e ICP-MS), a viscosidade é um parâmetro importante na representatividade das amostras, influenciando nos processos de formação e transporte do aerossol até o plasma. Quanto maior a viscosidade, maior a tensão superficial e, consequentemente, menor a eficiência de introdução da amostra para o plasma. ${ }^{42} \mathrm{~A}$ viscosidade da solução afeta a formação do spray no nebulizador, portanto o tamanho das gotas e sua separação na câmara de nebulização são prejudicadas. Soluções mais viscosas geram gotas maiores e em menor quantidade, o que evidencia a importância da calibração do equipamento de análise empregando solução a base do solvente extrator, garantindo a compatibilização da matriz. Dessa forma, evita-se a falsa conclusão de que a extração foi menos eficiente quando se emprega um solvente mais viscoso, quando na realidade o que ocorre é a introdução de um menor volume de solução, diminuindo a sensibilidade do método. ${ }^{42}$

Estudos descrevem que o teor de água acima de $50 \%\left(\mathrm{~m} \mathrm{~m}^{-1}\right)$ na síntese dos NADES dificulta significativamente a formação das interações intermoleculares de solventes eutéticos a base de cloreto de colina, levando à perda das suas propriedades físico-químicas. ${ }^{38}$ A ruptura das ligações de hidrogênio dos NADES leva à perda da eficiência de extração dos analitos em diferentes matrizes..$^{38,43}$ No presente estudo, os NADES foram sintetizadas usando 10 moles de água (aproximadamente $36 \% \mathrm{~m} \mathrm{~m}^{-1}$ ), resultando em solventes com menor viscosidade em comparação com outros baseados em semelhantes combinações de precursores, anteriormente apresentados na literatura. ${ }^{44,45}$

Devido aos seus precursores, os NADES apresentam elevados teores de carbono, o que pode ocasionar interferência de transporte ou ainda instabilidade no plasma, dependendo da quantidade de carbono presente. ${ }^{46,47}$ Além disso, já foi demonstrado que as reações de transferência de carga com as espécies de carbono ocasionam aumento da taxa de ionização de alguns analitos devido ao aumento da população de átomos e íons dos analitos em estado excitado, resultando em aumento da sensibilidade, em razão do aumento do sinal analítico. ${ }^{48}$

Após a síntese dos dois diferentes NADES buscou-se avaliar sua potencialidade para a extração de $\mathrm{As}, \mathrm{Ca}, \mathrm{Cd}, \mathrm{Cr}, \mathrm{Cu}, \mathrm{Fe}, \mathrm{K}, \mathrm{Mg}, \mathrm{Mn}$, $\mathrm{Na}, \mathrm{P}$ e Zn em amostras de materiais de referência de suplemento mineral e fosfato de rocha com o auxílio de ultrassom e banho por aquecimento. Os resultados obtidos foram comparados com o procedimento comparativo empregado (MW-AD) e com os valores descritos nos laudos dos materiais de referência. Nas Tabelas 3 e 4 estão apresentados os teores indicados nos laudos dos materiais de referência, os obtidos para os analitos avaliados empregando MW-AD e extração pelos diferentes NADES utilizados, empregando UAE e extração por banho de aquecimento para as amostras de fosfato de rocha e suplemento mineral, respectivamente.

Os limites de detecção (LOD) para os métodos de MW-AD, UAE e extração por aquecimento foram calculados considerando o desvio padrão de dez medições da solução do branco $\left(S_{\text {branco }}\right)$ e o valor da inclinação da curva de calibração (a), de acordo com a equação LOD $=3$. $\left(\mathrm{S}_{\text {branco }}\right) / \mathrm{a} \cdot{ }^{49,50}$ Para as análises no ICP OES os valores de LOD variaram de $1,61.10^{-2} \mathrm{mg} \mathrm{kg}^{-1}$ para Ca a $2,45.10^{-3} \mathrm{mg} \mathrm{kg}^{-1}$ para $\mathrm{Mg}$ enquanto que para as análises no ICP-MS os valores de LOD variaram de $1,68.10^{-3} \mathrm{mg} \mathrm{kg}^{-1}$ para As a $1,96.10^{-4} \mathrm{mg} \mathrm{kg}^{-1}$ para Cd.

Os resultados de recuperação obtidos empregando o método padrão (MW-AD) nas amostras de suplemento mineral foram de $88 \%$ (para $\mathrm{Ca}$ ) a $110 \%$ (para $\mathrm{Mn}$ ), com exceção do elemento $\mathrm{K}$ com $77 \%$ de concordância. Para as amostras de fosfato de rocha,

Tabela 3. Concentrações (média \pm desvio padrão, $\mathrm{n}=3$ ) de $\mathrm{As}, \mathrm{Ca}, \mathrm{Cd}, \mathrm{Cr}, \mathrm{Cu}, \mathrm{Fe}, \mathrm{K}, \mathrm{Mg}, \mathrm{Mn}, \mathrm{Na}, \mathrm{P}$ e Zn em amostras de fosfato de rocha (RM-Agro 2003a) empregando digestão ácida assistida por radiação micro-ondas (MW-AD), extração assistida por ultrassom (UAE) e extração em banho de aquecimento

\section{FOSFATO DE ROCHA}

\begin{tabular}{|c|c|c|c|c|c|c|c|c|c|c|c|}
\hline \multirow{3}{*}{ Analito } & \multirow{3}{*}{$\begin{array}{l}\text { Valor certificado } \\
\quad\left(\mathrm{mg} \mathrm{kg}^{-1}\right)\end{array}$} & \multirow{2}{*}{\multicolumn{2}{|c|}{ MW-AD }} & \multicolumn{4}{|c|}{ AC-AM ${ }^{a}$} & \multicolumn{4}{|c|}{ AC-Xil ${ }^{b}$} \\
\hline & & & & \multicolumn{2}{|l|}{ UAE } & \multicolumn{2}{|c|}{$\overline{\text { Banho de aquecimento }}$} & \multicolumn{2}{|c|}{ UAE } & \multicolumn{2}{|c|}{ Banho de aquecimento } \\
\hline & & $\begin{array}{l}\text { Valor obtido } \\
\left(\mathrm{mg} \mathrm{kg}^{-1}\right)\end{array}$ & $\mathrm{R} *(\%)$ & $\begin{array}{l}\text { Valor obtido } \\
\left(\mathrm{mg} \mathrm{kg}^{-1}\right)\end{array}$ & $\mathrm{R}^{*}(\%)$ & $\begin{array}{l}\text { Valor obtido } \\
\left(\mathrm{mg} \mathrm{kg}^{-1}\right)\end{array}$ & $\mathrm{R}^{*}(\%)$ & $\begin{array}{l}\text { Valor obtido } \\
\left(\mathrm{mg} \mathrm{kg}^{-1}\right)\end{array}$ & $\mathrm{R}^{*}(\%)$ & $\begin{array}{l}\text { Valor obtido } \\
\left(\mathrm{mg} \mathrm{kg}^{-1}\right)\end{array}$ & $\mathrm{R}^{*}(\%)$ \\
\hline As & $15,5 \pm 7,2$ & $14,5 \pm 0,73$ & 94 & $13,4 \pm 1,5$ & 86 & $9,4 \pm 0,56$ & 61 & $11,7 \pm 0,010$ & 75 & $5,9 \pm 1,7$ & 38 \\
\hline $\mathrm{Ca}$ & $36,0 \pm 5,6^{\mathrm{d}}$ & $30,5 \pm 1,7^{\mathrm{d}}$ & 85 & $27,1 \pm 2,6^{\mathrm{d}}$ & 75 & $22,6 \pm 5,0^{\mathrm{d}}$ & 63 & $22,9 \pm 1,7^{\mathrm{d}}$ & 64 & $19,9 \pm 1,7^{\mathrm{d}}$ & 55 \\
\hline $\mathrm{Cd}$ & $21,3 \pm 3,0$ & $16 \pm 0,7$ & 75 & $13,1 \pm 1,4$ & 61 & $11,8 \pm 0,81$ & 56 & $15,1 \pm 0,30$ & 71 & $14,2 \pm 0,090$ & 67 \\
\hline $\mathrm{Cr}$ & $288,8 \pm 18,9$ & $218,7 \pm 1,5$ & 76 & $286,4 \pm 2,4$ & 99 & $66,1 \pm 1,4$ & 23 & $236,1 \pm 1,9$ & 82 & $95,6 \pm 0,010$ & 33 \\
\hline $\mathrm{Cu}$ & $36,2 \pm 12,6$ & $36,9 \pm 0,10$ & 102 & $19,2 \pm 0,10$ & 53 & $19,8 \pm 0,030$ & 55 & $16,4 \pm 0,040$ & 45 & $14,8 \pm 0,0030$ & 41 \\
\hline $\mathrm{Fe}$ & $1708,6 \pm 62,1$ & $1360,6 \pm 0,10$ & 80 & $205,4 \pm 0,030$ & 12 & $301,1 \pm 0,10$ & 18 & $165,7 \pm 0,12$ & 10 & $204,9 \pm 0,020$ & 12 \\
\hline K & $567,9 \pm 51,7$ & $670,9 \pm 0,10$ & 118 & $263 \pm 0,1$ & 46 & $342 \pm 0,1$ & 60 & $211 \pm 0,1$ & 37 & $244 \pm 0,1$ & 43 \\
\hline $\mathrm{Mg}$ & $2695,9 \pm 132,5$ & $3064,3 \pm 0,10$ & 114 & $1567,7 \pm 0,70$ & 58 & $1534,9 \pm 0,20$ & 57 & $1333,2 \pm 0,14$ & 46 & $1377,5 \pm 0,090$ & 51 \\
\hline Mn & $16,3 \pm 6,9$ & $15,3 \pm 0,10$ & 94 & $6,2 \pm 0,020$ & 38 & $8,2 \pm 0,010$ & 50 & $4,5 \pm 0,020$ & 28 & $6,2 \pm 0,020$ & 38 \\
\hline $\mathrm{Na}$ & $5350,2 \pm 273,4$ & $5128,9 \pm 0,10$ & 94 & $2687,7 \pm 0,15$ & 50 & $2704,2 \pm 0,70$ & 51 & $2908,2 \pm 0,11$ & 54 & $2550,3 \pm 0,030$ & 48 \\
\hline $\mathrm{P}$ & $14,3 \pm 3,9^{\mathrm{d}}$ & $13,7 \pm 0,80^{\mathrm{d}}$ & 96 & $15,7 \pm 1,6^{\mathrm{d}}$ & 110 & $9,9 \pm 0,90^{\mathrm{d}}$ & 70 & $14,2 \pm 2,2^{\mathrm{d}}$ & 100 & $10,6 \pm 0,90^{\mathrm{d}}$ & 74 \\
\hline $\mathrm{Zn}$ & $277,9 \pm 12,8$ & $306,2 \pm 0,10$ & 110 & $288,9 \pm 0,010$ & 105 & $234,7 \pm 0,020$ & 85 & $265,3 \pm 0,010$ & 95 & $230,1 \pm 0,070$ & 83 \\
\hline
\end{tabular}

${ }^{\mathrm{a}} \mathrm{NADES}$ ácido cítrico, ácido málico e água; ${ }^{\mathrm{b}} \mathrm{NADES}$ ácido cítrico, xilitol e água; ${ }^{\mathrm{c}}$ Referências 33,34 ; ${ }^{\mathrm{d}} \mathrm{Ca}$ e P- Concentrações em \% $\mathrm{m} \mathrm{m}^{-1}$; $* \mathrm{R}$ - \% de concordância com o valor certificado. 
Tabela 4. Concentrações determinadas (média \pm desvio padrão, $\mathrm{n}=3$ ) de $\mathrm{Ca}, \mathrm{Cd}, \mathrm{Cu}, \mathrm{Fe}, \mathrm{K}, \mathrm{Mg}, \mathrm{Mn}, \mathrm{Na}$ e Zn em amostras de suplemento mineral (RM-Agro 2001a) empregando digestão ácida assistida por radiação micro-ondas (MW-AD), extração assistida por ultrassom (UAE) e extração em banho de aquecimento

\begin{tabular}{|c|c|c|c|c|c|c|c|c|c|c|c|}
\hline \multicolumn{12}{|c|}{ SUPLEMENTO MINERAL } \\
\hline \multirow{3}{*}{ Analito } & \multirow{3}{*}{$\begin{array}{l}\text { Valor certifica- } \\
\text { do }\left(\mathrm{g} \mathrm{kg}^{-1}\right)\end{array}$} & \multirow{2}{*}{\multicolumn{2}{|c|}{ MW-AD }} & \multicolumn{4}{|c|}{$\mathrm{AC}-\mathrm{AM}^{\mathrm{a}}$} & \multicolumn{4}{|c|}{$\mathrm{AC}-\mathrm{Xil}{ }^{\mathrm{b}}$} \\
\hline & & & & \multicolumn{2}{|l|}{ UAE } & \multicolumn{2}{|c|}{ Banho de aquecimento } & \multicolumn{2}{|l|}{ UAE } & \multicolumn{2}{|c|}{ Banho de aquecimento } \\
\hline & & $\begin{array}{c}\text { Valor obtido } \\
\left(\mathrm{g} \mathrm{kg}^{-1}\right)\end{array}$ & $\mathrm{R} *(\%)$ & $\begin{array}{l}\text { Valor obtido } \\
\qquad\left(\mathrm{g} \mathrm{kg}^{-1}\right)\end{array}$ & $\mathrm{R} *(\%)$ & $\begin{array}{l}\text { Valor obtido } \\
\qquad\left(\mathrm{g} \mathrm{kg}^{-1}\right)\end{array}$ & $\mathrm{R}^{*}(\%)$ & $\begin{array}{l}\text { Valor obtido } \\
\qquad\left(\mathrm{g} \mathrm{kg}^{-1}\right)\end{array}$ & $\mathrm{R} *(\%)$ & $\begin{array}{l}\text { Valor obtido } \\
\qquad\left(\mathrm{g} \mathrm{kg}^{-1}\right)\end{array}$ & $\mathrm{R} *(\%)$ \\
\hline $\mathrm{Ca}$ & $187,8 \pm 36,3$ & $166,7 \pm 0,70$ & 88 & $178,3 \pm 1,8$ & 97 & $152,2 \pm 1,5$ & 81 & $174,5 \pm 0,80$ & 93 & $137,9 \pm 1,0$ & 74 \\
\hline $\mathrm{Cd}$ & $425,3 \pm 76,4^{d}$ & $450,4 \pm 0,20^{\mathrm{d}}$ & 106 & $460,7 \pm 1,3^{\mathrm{d}}$ & 108 & $469,8 \pm 5,1^{\mathrm{d}}$ & 110 & $511,1 \pm 0,90^{\mathrm{d}}$ & 120 & $464,8 \pm 0,70^{\mathrm{d}}$ & 110 \\
\hline $\mathrm{Cu}$ & $803,8 \pm 86,5^{\mathrm{e}}$ & $775,9 \pm 0,040^{\mathrm{e}}$ & 97 & $473 \pm 1^{\mathrm{e}}$ & 58 & $463 \pm 1^{\mathrm{e}}$ & 58 & $448 \pm 1^{\mathrm{e}}$ & 56 & $436 \pm 1^{\mathrm{e}}$ & 54 \\
\hline $\mathrm{Fe}$ & $2729 \pm 213^{e}$ & $2914,1 \pm 0,20^{\mathrm{e}}$ & 107 & $331,9 \pm 0,050^{\mathrm{e}}$ & 12 & $451,7 \pm 0,070^{\mathrm{e}}$ & 17 & $332,2 \pm 0,090^{\mathrm{e}}$ & 12 & $331,2 \pm 0,20^{\mathrm{e}}$ & 12 \\
\hline $\mathrm{K}$ & $35,6 \pm 4,3$ & $27,3 \pm 1,3$ & 77 & $29,6 \pm 0,10$ & 83 & $28,9 \pm 0,10$ & 81 & $28,7 \pm 0,20$ & 81 & $28,9 \pm 0,30$ & 76 \\
\hline $\mathrm{Mg}$ & $10,8 \pm 2,7$ & $10,3 \pm 0,20$ & 95 & $6,5 \pm 0,20$ & 61 & $6,7 \pm 0,60$ & 62 & $6,6 \pm 1,2$ & 61 & $6,6 \pm 0,30$ & 61 \\
\hline $\mathrm{Mn}$ & $1571 \pm 141^{\mathrm{e}}$ & $1744,1 \pm 0,020^{\mathrm{e}}$ & 110 & $1775,1 \pm 0,10^{\mathrm{e}}$ & 113 & $1444,9 \pm 0,50^{\mathrm{e}}$ & 92 & $1820,2 \pm 0,30^{\mathrm{e}}$ & 116 & $1327,7 \pm 0,10^{\mathrm{e}}$ & 85 \\
\hline $\mathrm{Na}$ & $80 \pm 15$ & $78,9 \pm 0,30$ & 99 & $77,1 \pm 0,50$ & 96 & $77,2 \pm 0,60$ & 96 & $75,6 \pm 0,10$ & 95 & $70,2 \pm 0,40$ & 87 \\
\hline $\mathrm{P}$ & $84,5 \pm 17,3$ & $79,4 \pm 0,40$ & 96 & $97,1 \pm 0,50$ & 115 & $78,3 \pm 0,40$ & 93 & $100,2 \pm 0,60$ & 119 & $85,5 \pm 0,90$ & 101 \\
\hline
\end{tabular}

a NADES ácido cítrico, ácido málico e água; ${ }^{\text {a }} \mathrm{NADES}$ ácido cítrico, xilitol e água; ${ }^{\mathrm{c}}$ Referências 33,34 ; ${ }^{\mathrm{d}} \mathrm{Cd}-\mathrm{Concentrações} \mathrm{em} \mu \mathrm{g}$ kg-1; ${ }^{\mathrm{e}} \mathrm{Cu}$, Fe e Mn- Concentrações em mg kg${ }^{-1}$; *R- \% de concordância com o valor certificado.

a MW-AD apresentou concordância entre 80 e $110 \%$, exceto para $\mathrm{Cd}$ e $\mathrm{Cr}$ com 75 e $76 \%$ de recuperação, respectivamente. A Figura 1 apresenta esquematicamente os valores para os analitos avaliados, em porcentagem, para os métodos MW-AD, UAE e extração por banho de aquecimento, para as amostras avaliadas. Foram consideradas aceitáveis as recuperações na faixa de 80 a $110 \% .{ }^{49}$
Para o NADES AC-AM usando o método de UAE foram obtidas recuperações satisfatórias para As, $\mathrm{Cr}, \mathrm{P}$ e Zn em amostras de fosfato de rocha e de $\mathrm{Ca}, \mathrm{Cd}, \mathrm{K}$ e $\mathrm{Na}$ em amostras de suplemento mineral. Para o método de extração por banho de aquecimento utilizando o mesmo solvente extrator (NADES AC-AM), observou-se recuperações em um intervalo aceitável apenas para $\mathrm{Zn}$ em amostras

a) Fosfato de Rocha

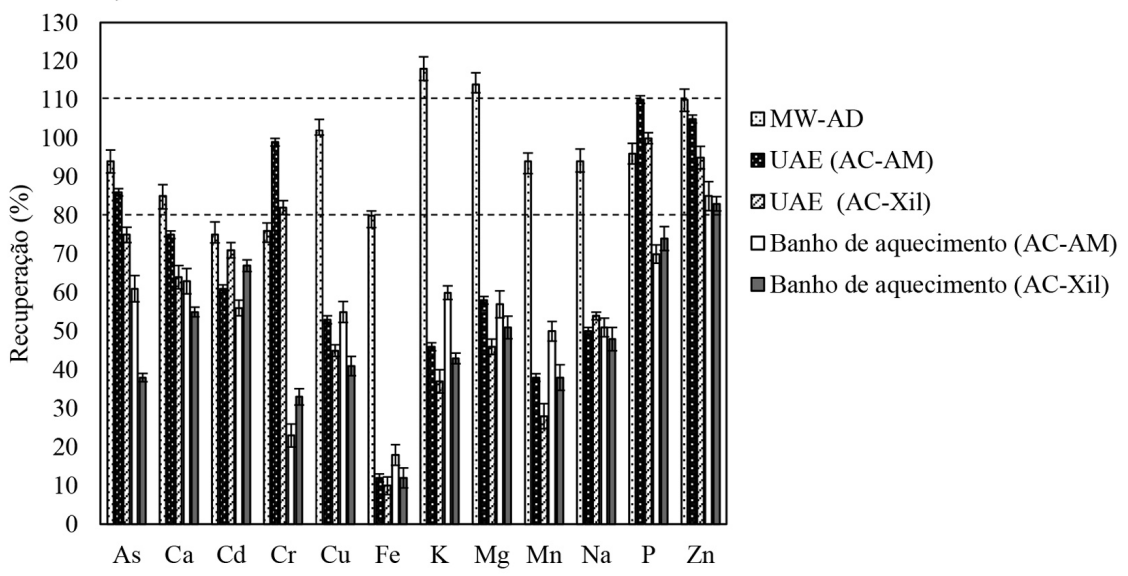

b) Suplemento Mineral

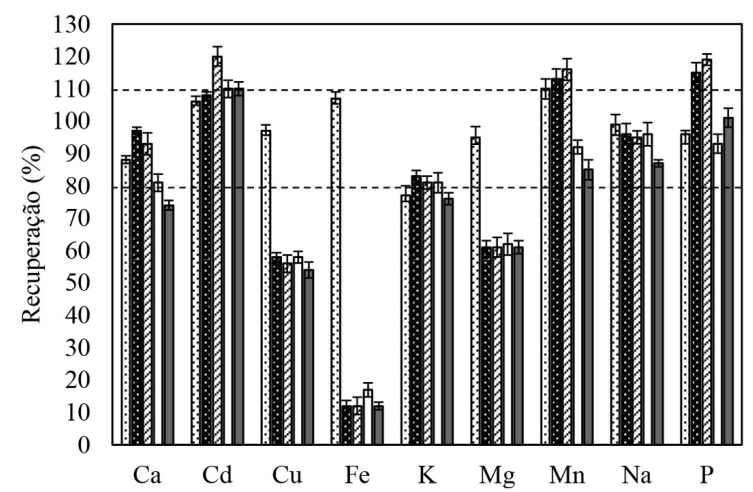

?MW-AD

- UAE (AC-AM)

$\square \operatorname{UAE}(\mathrm{AC}-\mathrm{Xil})$

$\square$ Banho de aquecimento (AC-AM)

$\square$ Banho de aquecimento (AC-Xil)

Figura 1. Valores de recuperação(\%) para $\mathrm{As}, \mathrm{Ca}, \mathrm{Cd}, \mathrm{Cr}, \mathrm{Cu}, \mathrm{Fe}, \mathrm{K}, \mathrm{Mg}, \mathrm{Mn}, \mathrm{Na}, \mathrm{P}$ e Zn para os métodos de digestão ácida assistida por radiação micro-ondas (MW-AD), extração assistida por ultrassom (UAE) e extração por banho de aquecimento empregando NADES ácido cítrico, ácido málico e água (AC-AM) e NADES ácido cítrico, xilitol e água (AC-Xil) para as amostras referência a) fosfato de rocha e b) suplemento mineral ${ }^{33,34}$ 
de fosfato de rocha e para $\mathrm{Ca}, \mathrm{Cd}, \mathrm{K}, \mathrm{Mn}, \mathrm{Na}$ e $\mathrm{P}$ em amostras de suplemento mineral.

O método UAE utilizando NADES AC-Xil apresentou recuperações no intervalo aceitável para $\mathrm{Ca}, \mathrm{K}$ e $\mathrm{Na}$ em amostras de suplemento mineral, enquanto para as amostras de fosfato de rocha para os elementos $\mathrm{Cr}$, P e Zn. Porém, para o método de extração em aquecimento com o mesmo NADES, as recuperações variaram de 85 a $110 \%$ para os elementos $\mathrm{Cd}, \mathrm{Mn}, \mathrm{Na}$ e $\mathrm{P}$ nas amostras de suplemento mineral. No caso das amostras de fosfato de rocha utilizando a técnica de extração com aquecimento, foi obtida recuperação satisfatória para $\mathrm{Zn}$.

Em um contexto geral, as extrações por UAE apresentaram melhores resultados quando comparadas com as extrações por banho de aquecimento. De fato, a UAE tem se mostrado uma técnica efetiva para extração de uma grande parcela de analitos e em diferentes tipos de amostras, devido ao fenômeno de cavitação que favorece a transferência de calor. ${ }^{15,16}$

Os resultados obtidos pelos métodos propostos foram comparados com os valores de concentração determinados pelo método de referência (MW-AD), sendo observadas diferenças significativas ao nível de $95 \%$ de confiança (teste $t$ de Student) para alguns dos casos. A comparação desses resultados revelou diferenças significativas para alguns elementos, sugerindo diferentes interações químicas entre os NADES sintetizados e cada elemento avaliado. ${ }^{30} \mathrm{Na}$ extração das amostras de fosfato de rocha, os melhores resultados foram para As (UAE e AC-AM), Cr (UAE e AC-AM), P (UAE e AC-Xil) e Zn (UAE e AC-Xil; banho de aquecimento e AC-Xil). Em relação ao suplemento mineral, não foram observadas diferenças significativas para Ca (UAE e AC-AM; UAE e AC-Xil), Cd (UAE e AC-AM; banho de aquecimento e AC-Xil), K (UAE e AC-AM; banho de aquecimento e AC-Xil), Mn (UAE e AC-AM; banho de aquecimento e AC-Xil), $\mathrm{Na}$ (UAE e AC-AM; banho de aquecimento e AC-Xil; banho de aquecimento e AC-AM) e $\mathrm{P}$ para todos os métodos avaliados.

Os dois solventes sintetizados (AC-AM e AC-Xil) foram eficientes para extração simultânea de $\mathrm{Cd}, \mathrm{Mn}, \mathrm{Na}$ e $\mathrm{P}$ em amostras de suplemento mineral e $\mathrm{Zn}$ em amostras de fosfato de rocha, empregando ambos os métodos de extração propostos. Porém, para a maioria dos analitos foram observadas baixas recuperações (de 10 a 77\%) para ambas as amostras. Um ponto a ser destacado é a capacidade dos NADES extraírem alguns analitos e outros não. Fica evidente a seletividade que, a depender das exigências e objetivo da análise, poderão ter sua formulação adequada para os diferentes tipos de amostras. Da mesma forma, as condições de extração também podem ser otimizadas, considerando as variáveis envolvidas como tempo, temperatura, dentre outras.

É válido ressaltar que até o presente momento não há relato na literatura de aplicação dessa classe de solventes em matrizes inorgânicas. Estudos mostraram que os NADES não são considerados solventes universais, pelo contrário, são solventes altamente seletivos e moduláveis. ${ }^{43}$ Isso sugere que a escolha de um método de síntese dos NADES e a proporção de seus reagentes também deve levar em consideração a matriz e o analito de interesse, pois a existência de efeitos matriciais pode inibir a extração completa de alguns analitos, ${ }^{43}$ fato comprovado com os resultados obtidos no presente estudo. Outra potencialidade vislumbrada é o emprego de NADES em estudos de especiação química, em que NADES adequadamente sintetizados teriam a capacidade de se ligar aos analitos que estão ligados de forma específica na matriz.

O método da Eco-Scale analítica foi empregado para avaliar o quão ambientalmente corretas são as metodologias analíticas propostas. Para isso, são atribuídos pontos de penalidades para parâmetros que não estão de acordo com os princípios da química verde, no qual são considerados o gasto energético, volume e segurança do solvente empregado e a geração de resíduo. ${ }^{43}$ As pontuações da Eco-Scale analítica foram 75 por amostra para MW-AD e 97 para os métodos UAE e extração por banho de aquecimento. Nessa abordagem, uma pontuação $>75$ representa uma excelente análise verde, enquanto $75>$ x > 50 representa uma análise verde aceitável. ${ }^{51}$ A pontuação mais baixa do método de MW-AD está relacionada à parâmetros como maior gasto energético, formação de vapor ácido, maior volume de resíduo e a segurança dos reagentes empregados no procedimento.

\section{CONCLUSÕES}

Neste trabalho, dois NADES foram preparados a partir da combinação entre ácido málico, ácido cítrico, xilitol e água e, posteriormente, avaliados como possíveis solventes extratores de matrizes inorgânicas. A extração assistida por radiação ultrassônica (UAE) e a extração por banho de aquecimento mostraram-se como métodos eficientes para a extração seletiva dos analitos monitorados em amostras de fertilizante fosfatados e suplemento mineral. Os NADES se mostraram solventes seletivos, interagindo de maneira diferente com cada analito estudado em matrizes complexas. Não foi possível identificar um solvente ou método de extração ideal para determinação quantitativa de todos analitos simultaneamente nas matrizes avaliadas. Deve ser ressaltada a dificuldade existente na solubilização dos materiais inorgânicos avaliados, altamente refratários. Os resultados mostraram que os NADES são promissores para a extração de matrizes inorgânicas, além de serem compatíveis com técnicas com fonte de plasma. Além disso, os métodos propostos apresentaram-se em consonância aos princípios da Química Verde, exibindo vantagens como simplicidade operacional, uso de reagentes não voláteis e emprego de menores volumes de solvente extrator. Portanto, pode ser considerada uma alternativa aos métodos convencionais de preparo de amostras inorgânicas, como fosfato de rocha e suplemento mineral.

\section{AGRADECIMENTOS}

Os autores agradecem o auxílio financeiro recebido da FAPESP (Projetos 2015/08893-4, 2015/14488-0, 2016/17304-0, 2017/18531-3 e 2019/22113-8) e do Instituto Nacional de Tecnologias Alternativas para Detecção, Avaliação Toxicológica e Remoção de Micropoluentes e Radioativos - INCT - DATREM (Fapesp - 14/509454 e CNPq 465571/2014-0). A.R.A. Nogueira agradece ao CNPq (308178/20181). A.P.R. Santana e T.G.S. Guimarães agradecem à CAPES (Código de Financiamento 001) pela bolsa concedida e ao PPGQuímica/ UNESP-SJRP.

\section{REFERÊNCIAS}

1. Plotka-Wasylka, J.; Rutkowska, M.; Owczarek, K.; Tobiszewski, M.; Namiesnik, J.; TrAC - Trends Anal. Chem. 2017, 91, 12.

2. Pacheco- Fernandez, I.; Pino, V. Curr. Opin. Green Sustain. Chem. 2019, $18,42$.

3. Rocha, D. L.; Batista, A. D.; Rocha, F. R. P.; Donati, G. L.; Nóbrega, J. A.; TrAC - Trends Anal. Chem. 2013, 45, 79.

4. Tobiszewski, M.; Mechlińska, A.; Zygmunt, B.; Mamieśnik, J.; TrACTrends Anal. Chem. 2009, 28, 943.

5. Abbott, A. P.; Capper, G.; Davies, D. L.; Rasheeda, R. K.; Tambyrajah, V.; Chem. Commun. 2003, 1, 70.

6. Choi, Y. H.; van Spronsen, J.; Dai, Y.; Verberne, M.; Hollmann, F.; Arends, I. W. C. E.; Witkamp, G-J.; Verpoorte, R.; Plant Physiol. 2011, 156, 1701.

7. Dai, Y.; van Spronsen, J.; G. J. Witkamp, Verpoorte, R.; Anal. Chim. Acta 2013, 766, 61. 
8. Abo-Hamad, A.; Hayyan, M.; Alssadi, M. A.; Hashim, M. A.; Chem. Eng. J. 2015, 273, 551.

9. Fernández, M. A.; Boiteux, J.; Espino, M.; Gomez, F. J. V.; Silva, M. F.; Anal. Chim. Acta 2018, 1038, 1.

10. Espino, M.; M. A. Fernández, Gomez, F. J. V.; Silva, M. F.; $\operatorname{Tr} A C-$ Trends Anal. Chem. 2016, 76, 126.

11. Moraes, D. P.; Bizzi, C. A.; Nóbreega, J. A.; Flores, E. M. M.; Nogueira, A. R. A.; Barin, J. S.; Mesko, M. F. Em Métodos de Preparo de Amostras para Análise Elementar; Krug, F. J., Rocha, F. R. P., eds.; EditSBQ: São Paulo, 2019, cap. 10.

12. Barin, J. S.; Bizzi, C. A.; Flores, E. M. M.; Nóbrega, J. A.; Krug, F. J. Em Métodos de Preparo de Amostras para Análise Elementar; Krug, F. J.; Rocha, F. R. P., eds.; EditSBQ: São Paulo, 2019, cap. 9.

13. Matusiewicz, H. Em Comprehensive Analytical Chemistry; Mester, Z.; Sturgeon, R., eds.; Elsevier: Amsterdam, 2003, cap. 6.

14. Bancroft, W. D.; Murphy, N. F.; J. Phys. Chem. 1935, 3, 377.

15. Rutkowska, M.; Namieśnik J.; Konieczka, P. Em The Application of Green Solvents in Separation Processes; Pena-Pereira, F.; Tobiszewski. M., eds.; Elsevier: Amsterdam, 2017, cap. 10.

16. Vinatoru, M.; Mason, T. J.; Calinescu, I.; TrAC - Trends Anal. Chem. 2017, 97, 159.

17. Chemat, F.; Rombaut, N.; Sicarie, A-G.; Meullemiestre, A.; FabianoTixier, A-S.; Albert-Vian, M.; Ultrason. Sonochem. 2017, 34, 540.

18. Lima, E. C.; Barbosa, F.; Krug, F. J.; Silva, M. M.; Vale, M. G. R.; J. Anal. At. Spectrom. 2000, 15, 995.

19. Teixeira, L. S.; Vieira, H. P.; Windmoller, C. C.; Nascentes, C. C.; Talanta 2014, 119, 232.

20. Canapari, S.; Cardarelli, E.; Ghichi, S.; Scimonelli, L.; Talanta 2005, 66, 1122.

21. López-García, I.; Campillo, N.; Arnau-Jerez, I.; Hernández-Córdoba M.; Anal. Chim. Acta 2005, 531, 125.

22. El-Sheikh, A. H.; Al-Degs, Y. S.; Sweileh, J. A.; Said, A. J.; Talanta 2013, 116, 482.

23. Abdel-Haleem, A. S.; Sroor, A.; El-Bahi, S. M.; Zohny, E.; Appl. Radiat. Isot. 2001, 55, 569.

24. Mortvedt, J. J.; J. Environ. Qual. 1987, 16, 137.

25. Soetan, K. O.; Olaiya, C. O.; Oyewole, E.; Afr. J. Food Sci. 2010, 4, 200.

26. Gonçalves, J. R.; Gonçalves, R. M.; Gonçalves, R. M.; Revista Processos Químicos 2010, 4, 68.

27. Tokarnia, C. H.; Dobereiner, J.; Peixoto, P. V.; Pesquisa Veterinária Brasileira 2000, 20, 127.

28. Marçal, W. S.; Gaste, L.; Liboni, M.; Pardo, P. E.; Nascimento, M. R.; Hisasi, C. S.; Vet. Arch. 1999, 69, 355.
29. Shishov, A.; Bulatov, A.; Locatelli, M.; Carradori, S.; Andruch, V.; Microchem. J. 2017, 135, 33.

30. Santana, A. P. R.; Andrade, D. F.; Mora-Vargas, J. A.; Amaral, C. D. B.; Oliveira, A.; Gonzalez, M. H.; Talanta 2019, 199, 361.

31. Santana, A. P. R.; Andrade, D. F.; Guimarães, T.G.S.; Amaral, C. D. B.; Oliveira, A.; Gonzalez, M. H.; Talanta 2020, 216, 120956.

32. Montaser, A.; Golightly, D. W.; Inductively Coupled Plasmas in Analytical Atomic Spectrometry; Wiley-VCH: New York, USA, 1998.

33. Nogueira, A. R. A.; Souza, G. B.; Bossu, C. M.; Bianchi, S. R.; Verhalen, T. R.; Silva, P. T.; Peixoto, A. A. J.; Silva, C. S.; J. Phys.: Conf. Ser. 2016, 733, 012005.

34. Silva, C. S.; Souza, G. B.; Nogueira, A. R. A.; Accredit. Qual. Assur. 2018, 23, 39.

35. Santana, A. P. R.; Mora-Vargas, J. A.; Guimarães, T.G.S.; Amaral, C. D. B.; Oliveira, A.; Gonzalez, M. H.; J. Mol. Liq. 2019, 293, 111452.

36. Nam, M. W.; Zhao, J.; Lee, M. S.; Jeong, J. H.; Lee, J.; Green Chem. 2015, 17, 1718

37. Kane, P. F.; Hall, W. L. J. AOAC Int. 2006, 89, 1447.

38. Dai, Y.; Witkamp, G. J.; Verpoorte, R.; Choi, Y. H.; Food Chem. 2015, 187, 14.

39. Ribeiro, B. D.; Florindo, C.; Iff, L. C.; Coelho, M. A. Z.; Marrucho, I. M.; ACS Sustain. Chem. Eng. 2015, 3, 2469.

40. Dwamena, A. K.; Separations 2019, 6, 9.

41. Huang, Y.; Fend, F.; Jiang, J.; Qiao, Y.; Wu, T.; Voglmeir, J.; Chen, Z. G.; Food Chem. 2017, 221, 1400.

42. Thomas, R.; Practical Guide to ICP-MS: A Tutorial for Beginners, $2^{\text {nd }}$ ed., CRC Press: New York, 2013.

43. Gonzalez, C. G.; Choi, Y. H.; Verpoorte, R. Em Liquid-Phase Extraction; Poole, C. F., eds.; Elsevier: Amsterdam, 2020, cap. 19.

44. Atmamash, T.; Nasser, M. S.; Elhamarnah, Y.; Magzoub, M.; Ullah, R.; Qiblawey, H.; Aparicio, S.; Atilhan, M.; J. Mol. Liq. 2018, 256, 286.

45. Craveiro, R.; Aroso, I.; Flammia, V.; Carvalho, T.; Viciosa, M. T.; Dionísio, M.; Barreiros, S.; Reis R. L.; Duarte, A. R. C.; Paiva, A.; J. Mol. Liq. 2016, 215, 534.

46. Evans, E. H.; Giglio, J. J.; J. Anal. At. Spectrom. 1993, 8, 1.

47. Grindlay, G.; Mora, J.; De Loos-Vollebregt, M.; Vanhaecke, F.; Spectrochim. Acta, Part B 2013, 86, 42.

48. Grindlay, G.; Gras, L.; Mora, J.; De Loos-Vollebregt, M. T. C.; Spectrochim. Acta, Part B 2008, 63, 234.

49. http://www.inmetro.gov.br/Sidoq/Arquivos/CGCRE/DOQ/DOQCGCRE-8_05.pdf, acessada em janeiro 2021.

50. http://goldbook.iupac.org/PDF/goldbook.pdf, acessada em janeiro 2021.

51. Gałuszka, A.; Migaszewski, Z. M.; Konieczka, P.; Namieśnik, J.; TrAC - Trends Anal. Chem. 2012, 37, 61. 